\title{
Video Article \\ Minimally Invasive Muscle Embedding (MIME) - A Novel Experimental Technique to Facilitate Donor-Cell-Mediated Myogenesis
}

\author{
Joseph A Roche ${ }^{1}$, Morium Begam ${ }^{1}$, Sujay S Galen ${ }^{1}$ \\ ${ }^{1}$ Department of Health Care Sciences, Physical Therapy Program, College of Pharmacy and Health Sciences, Wayne State University
}

Correspondence to: Joseph A Roche at joseph.roche@wayne.edu

URL: https://www.jove.com/video/55731

DOI: doi:10.3791/55731

Keywords: Medicine, Issue 126, Skeletal muscle, muscle regeneration, muscle grafting, satellite cells, myogenesis, muscle disease, minimally invasive muscle embedding

Date Published: 8/24/2017

Citation: Roche, J.A., Begam, M., Galen, S.S. Minimally Invasive Muscle Embedding (MIME) - A Novel Experimental Technique to Facilitate DonorCell-Mediated Myogenesis. J. Vis. Exp. (126), e55731, doi:10.3791/55731 (2017).

\section{Abstract}

Skeletal muscle possesses regenerative capacity due to tissue-resident, muscle-fiber-generating (myogenic) satellite cells (SCs), which can form new muscle fibers under the right conditions. Although SCs can be harvested from muscle tissue and cultured in vitro, the resulting myoblast cells are not very effective in promoting myogenesis when transplanted into host muscle. Surgically exposing the host muscle and grafting segments of donor muscle tissue, or the isolated muscle fibers with their SCs onto host muscle, promotes better myogenesis compared to myoblast transplantation. We have developed a novel technique that we call Minimally Invasive Muscle Embedding (MIME). MIME involves passing a surgical needle through the host muscle, drawing a piece of donor muscle tissue through the needle track, and then leaving the donor tissue embedded in the host muscle so that it may act as a source of SCs for the host muscle. Here we describe in detail the steps involved in performing MIME in an immunodeficient mouse model that expresses a green fluorescent protein (GFP) in all of its cells. Immunodeficiency in the host mouse reduces the risk of immune rejection of the donor tissue, and GFP expression enables easy identification of the host muscle fibers (GFP+) and donor-cell-derived muscle fibers (GFP-). Our pilot data suggest that MIME can be used to implant an extensor digitorum longus (EDL) muscle from a donor mouse into the tibialis anterior (TA) muscle of a host mouse. Our data also suggest that when a myotoxin (barium chloride, $\mathrm{BaCl}_{2}$ ) is injected into the host muscle after MIME, there is evidence of donor-cell-derived myogenesis in the host muscle, with approximately $5 \%, 26 \%, 26 \%$ and $43 \%$ of the fibers in a single host TA muscle showing no host contribution, minimal host contribution, moderate host contribution, and maximal host contribution, respectively.

\section{Video Link}

The video component of this article can be found at https://www.jove.com/video/55731/

\section{Introduction}

Healthy skeletal muscle, even though post-mitotic, possesses excellent regenerative capacity due to the presence of tissue-resident myogenic cells known as satellite cells (SCs) ${ }^{1,2}$; and reviewed in ${ }^{3,4}$. However, under pathological conditions caused by muscular dystrophies, trauma or accelerated aging, the muscle regeneration might not keep up with muscle breakdown, and thus, progressive muscle fiber loss occurs ${ }^{5}$. Although effective methods have been developed to isolate SCs from muscle and expand them in culture to generate large numbers of myoblasts (and subsequently myotubes), attempts to generate physiologically relevant numbers of muscle fibers in host muscle have yielded only minimal success $^{6}$. As with many other cell types, when myoblasts are injected alone into host tissue, most of the cells do not engraft ${ }^{7,8}$. We have shown that neuromuscular electrical stimulation (NMES) facilitates donor-cell-derived myogenesis in regeneration-deficient host mouse muscle that was injected with human myoblasts ${ }^{8}$. Others have demonstrated that surgically grafting biopsied muscle or single muscle fibers with attached SCs, facilitate moderate myogenesis even without NMES, suggesting that implanting whole muscle fibers with SCs might be more advantageous than implanting myogenic cells alone ${ }^{9,10}$. Since donor or engineered muscle tissue grafted into host muscle produces better results than transplanting cells alone, it is possible that tissue or tissue-like structures might provide crucial cues for donor-cell engraftment; a concept which is becoming increasingly evident in cell-therapy studies involving various cell types ${ }^{10,11,12}$.

Recent data suggest that SCs obtained from humans more than 2 weeks post mortem generate myotubes in culture ${ }^{13}$. We therefore intend to assess if implantation of muscle tissue harvested post-mortem into a living host will reverse muscle fiber loss. We have developed a novel technique called MIME to implant donor muscle tissue into skeletal muscle tissue of a living host in order to promote donor-cell-derived myogenesis. MIME involves passing a surgical needle through the host muscle to create a needle track; drawing a small segment of donor muscle tissue through the needle track; leaving the donor tissue embedded in the host muscle; and closing the needle holes with tissue adhesive. After practicing the technique in euthanized mice studied in other experiments, we have now performed MIME in live mice that are immunodeficient and ubiquitously express a green fluorescent protein (GFP), and follow-up at 3 and 14 days post-MIME. At 3 days post-MIME, we confirm that donor mouse (GFP-) EDL muscle implanted into host mouse (GFP+) TA muscle, remains embedded in the host muscle. At 14 days post-MIME, after $\mathrm{BaCl}_{2}$ myotoxin injury to induce damage and myogenesis, we confirm that approximately $5 \%, 26 \%, 26 \%$ and $43 \%$ of the fibers in a single host TA muscle show no host contribution, minimal host contribution, moderate host contribution, and maximal host contribution, 
respectively. Central nucleation (a marker of degeneration and subsequent regeneration) is seen in approximately $95 \%$ of TA muscle fibers after MIME and myotoxin injection.

We study TA muscles 14 days after $\mathrm{MIME}+\mathrm{BaCl}_{2}$ because this time point captures the intermediate stage of regeneration, when a majority of the regenerated fibers are centrally nucleated. We study GFP+ mice as hosts for MIME, so that when we eventually transplant human cadaveric muscle into host mice, we will be able to easily distinguish muscle fibers of host and donor origin. We use the mouse EDL muscle as the experimental donor tissue, since single fibers from this muscle have shown greater myogenic potential than TA muscles ${ }^{9}$. The EDL muscle is also synergistic to the TA muscle and has similar fiber-type composition. Our preliminary data suggest that MIME is capable of facilitating donorcell-derived myogenesis in the host muscle.

\section{Protocol}

All studies involving live animals are approved by the Institution Animal Care and Use Committee (IACUC) at Wayne State University, Detroit, Michigan, USA, and are in accordance with the Guide for the Care and Use of Laboratory Animals (8th Edition, 2011, published by National Academies Press, 500 Fifth Street, NW, Lockbox 285, Washington, DC 20055, USA). As per the approved IACUC protocols, procedures involving pain and/or distress were performed under general anesthesia, which is induced and maintained by isoflurane inhalation (1.5 - $5 \%$ to effect). Anesthesia was verified by lack of withdrawal to toe pinch, and lack of palpebral or vibrissae responses (the percentage of isoflurane was increased as needed to maintain effect). Due to the minimally invasive nature of the protocols, a "sterile tips" technique was followed. While animals were under anesthesia, petroleum jelly was applied to the eyes to prevent dryness. No special treatments were required; however, diet gel was provided to the animals for $24 \mathrm{~h}$ following procedures that involved general anesthesia. The investigator is approved by the IACUC to withhold analgesia following MIME, since the procedure does not involve surgically exposing the host muscle, because it is confined to one hindlimb and does not affect normal function, and because many common analgesic drugs are known to affect normal muscle regeneration.

\section{Animal Models}

\section{Host mice for MIME}

1. Use NSG-GFP mice ( 8 - 10 week, male) as hosts for the muscle grafts studies.

NOTE: These mice are ideal hosts for allogeneic muscle grafts because they lack mature $T$ and $B$ lymphocytes and functional natural killer cells, are deficient in cytokine signaling, and have been used by others for allografting or xenografting non-muscle cells ${ }^{14}$. Ubiquitous GFP expression allows for easy identification of host (GFP+) and donor-cell-derived (GFP-) muscle fibers.

\section{Donor mice for MIME}

1. Use C57BL/6J mice (12 - 16 week) as donor mice. NOTE: These mice are suitable donor mice because they have a fully mapped genome, are not known to have any skeletal muscle pathology, are easily available and economical, and do not express GFP.

\section{Preparing Donor Muscle Tissue}

\section{Tying Guiding Sutures and Harvesting donor EDL muscles}

1. Euthanize the donor mice by cervical dislocation and thoracotomy performed under general anesthesia administered by a tabletop isoflurane vaporizer driven by medical oxygen (inhaled isoflurane; 2 - $5 \%$ to effect).

2. To access the EDL muscles, use a pair of surgical scissors to open the skin over the anterior surface of the leg. Locate the TA muscle in the anterior leg and remove it to visualize the EDL muscle that is situated behind the TA muscle. Slide the tip of a pair of forceps behind the EDL muscle and apply gentle traction to see if the toes extend (this confirms that the muscle is the EDL).

NOTE: Donor tissue area should be surgically, aseptically prepped prior to harvest.

3. Use $\sim 10 \mathrm{~cm}$ segments of 4-0 silk, braided, non-absorbable, sterile suture, and make two double knots to apply the guiding sutures to the proximal and distal tendons of the EDL muscle. (Figure 1A).

4. Cut the distal EDL tendon, reflect the EDL muscle and cut the proximal tendon.

5. Place the donor EDL muscles in a Petri dish filled with mouse Ringer solution.

\section{Preparing Host Mice for MIME}

\section{Anesthesia}

1. Place the host mouse under general anesthesia (inhaled isoflurane; 1.5 - $5 \%$ to effect) administered by a tabletop isoflurane vaporizer driven by medical oxygen.

2. Induce anesthesia in an induction chamber and then transfer the mouse to a nose-cone to maintain the anesthesia while performing other procedures on the animal. Provide thermal support with an isothermal gel heating pad while the animal is under anesthesia.

\section{Skin preparation}

1. To remove the animal's fur, apply a depilatory cream over the anterior aspect of the left hind leg. The right leg serves as a control leg for subsequent procedures.

2. Leave the depilatory cream on for $2 \mathrm{~min}$. Clean off the depilatory cream along with the detached fur with wipes soaked in phosphate buffered saline (PBS). After fur removal, scrub the skin with three alternating wipes of povidone-iodine scrubbing solution and $70 \%$ ethanol. 
3. Creating a needle track in the host TA muscle

1. Pass an 18-gauge (1 in long) surgical needle through the center of the host TA muscle along the muscle's long axis (Figure 1B). Pass the needle in a cephalo-caudal direction, along the length of the TA muscle, and through the center of the muscle belly (Figure 1B). NOTE: The purpose of this step is to create a needle track, into which a piece of donor muscle may be embedded. Embedding the donor tissue in the center of the TA muscle would potentially allow the SCs from the donor tissue to migrate and facilitate myogenesis across the entire host TA muscle.

4. Embedding donor tissue in the needle track of host TA muscle

1. Once the surgical needle is within the host TA muscle, pass the guiding sutures at one end of the donor tissue through the lumen of surgical needle in a caudo-cephalic direction (Figure 1C).

2. Draw the donor tissue through the needle track as the needle is withdrawn from the host TA muscle in a caudo-cephalic direction.

3. Use the guiding sutures at the caudal and cephalic ends of the donor tissue to adjust the placement of the donor tissue. Ensure that the donor tissue is ensconced within the host TA muscle (Figure 1D).

4. Once the donor tissue placement is optimized, cut off the guiding sutures, make any final adjustments to the donor tissue placement with fine-tipped forceps.

5. Seal cutaneous needle wounds (Figure 1E - F) by approximating the wound edge with forceps and applying veterinary tissue adhesive with the tip of a 27 -gauge surgical needle.

\section{Intramuscular Myotoxin Injection to Induce Concerted Muscle Degeneration and Regeneration}

1. After MIME, inject $50-60 \mu \mathrm{L}$ of $1.2 \% \mathrm{BaCl}_{2}$ (myotoxin) into the host TA muscle to induce extensive muscle fiber damage in the host muscle and the donor tissue embedded within it $^{15}$.

1. Inject $\mathrm{BaCl}_{2}$ at 3 sites along the length of the host TA muscle (proximal, middle and distal one-third of the muscle belly). NOTE: Injecting myotoxins like $\mathrm{BaCl}_{2}$, cardiotoxins and notexin into skeletal muscle induces concerted muscle fiber damage followed by regeneration ${ }^{16}$.

\section{Post-procedural Animal Care}

1. After MIME and $\mathrm{BaCl}_{2}$ injection, clean the host mouse's left hind leg with ethanol and apply a thin layer of petroleum jelly to protect the skin.

2. After post-procedural skin care, remove the host mouse from the nose cone.

3. Place the mouse in a solitary recovery cage without bedding. Provide thermal support to the recovering animal through an isothermal gel heating pad placed under half of the recovery cage.

NOTE: One half is not heated so that animal may move away from the heating pad if needed.

4. After the host mouse has completely recovered from anesthesia, return the animal to its original cage. Place the animal back in the animal facility until follow-up experiments are performed. Monitor the host mouse daily until it is ready for follow-up - example: at 3 or 14 days postMIME. Following MIME, animals are monitored by laboratory personnel as well by veterinary staff - this mainly involves assessing if animals are bright, alert and responsive; and also assessing if animals are showing overt signs of pain or are having difficulty moving, feeding, drinking and grooming.

\section{Tissue Collection}

\section{Harvesting host muscle}

1. Euthanize the host mice by cervical dislocation and thoracotomy performed under general anesthesia administered by a tabletop isoflurane vaporizer driven by medical oxygen (inhaled isoflurane; 2 - 5\% to effect). The investigator also has IACUC approval to harvest TA muscles under general anesthesia prior to euthanasia.

2. To access the host TA muscles, use a pair of surgical scissors to open the skin over the anterior surface of the leg. Locate the TA muscle in the anterior leg, cut the distal tendon, reflect the muscle and cut the proximal muscle attachments (see section 2.1).

3. Collect both left (experimental) and right (control) TA muscles.

\section{Snap freezing harvested muscles}

1. Weigh the harvested muscles by placing on weigh paper and weighing scale.

2. Briefly dip the muscles in mineral oil for cryoprotection. Blot off the excess oil.

3. Place muscles on aluminum foil, and snap freeze the muscles by rapidly immersing them in liquid nitrogen filled in a Dewar. Transfer the samples to labeled cryovials and store the samples in a $-80^{\circ} \mathrm{C}$ freezer for later studies.

\section{Histological Studies}

1. Cryosectioning muscle tissue to collect serial cross sections

1. Transfer the samples from the $-80^{\circ} \mathrm{C}$ freezer to a cryostat by placing them in a Dewar containing liquid nitrogen.

2. In the cryostat, handle one sample at a time. With a cold razor blade (blade stored in cryostat), cut the sample twice at the mid-belly of the TA muscle to produce a segment of muscle that is about $1-2 \mathrm{~mm}$ thick. Keep this segment for making the cryostat sections and return the remaining portions of muscle to its cryovial.

3. With the optimum cutting temperature (OCT) freezing compound, secure the thick segment of tissue onto a cryostat specimen disc. Place the specimen disc on the specimen holder. 
4. Rough-face the sample by cutting $20 \mu \mathrm{m}$ sections. Collect a few $20 \mu \mathrm{m}$ sections on glass slides for the cryostat sections.

5. If the quality of the $20 \mu \mathrm{m}$ sections is satisfactory, change the cutting thickness to $5 \mu \mathrm{m}$ and collect a few sections to assess the quality. If the $5 \mu \mathrm{m}$ sections are satisfactory in quality, collect serial sections. Collect $2-3$ sections per slide and collect enough sections for 3 slides.

6. Collect sections from both the experimental (left) and control (right) TA muscles of each animal.

\section{Studying GFP expression}

NOTE: Designate one set of the prepared slides (section 7.1) to study GFP expression in the muscle fibers.

1. Apply $\sim 50 \mu \mathrm{L}$ of anti-fade medium on the sections and apply a glass coverslip. Seal the coverslip edges with clear nail polish.

2. Using the light and fluorescent microscope (see Table of Materials), study the sections with a 10X objective and an appropriate filter for visualizing GFP.

1. Collect digital images $(\sim 15)$ of overlapping visual fields to cover the entire cross section of the TA muscle. Collect the phase contrast images for each of the visual fields by switching from fluorescence to phase contrast mode on the microscope.

3. Open images with a suitable imaging software that can tile individual images to produce a single composite image; e.g., use the "photomerge" option under File Menu in the imaging software listed in the Table of Materials.

4. Open the GFP fluorescence images with a suitable image analysis software (e.g., ImageJ). Circle individual muscle fibers using the "ROI tool" and record the Mean Fluorescence Intensity for each fiber.

1. Calculate the Maximum GFP (Fluorescence) Intensity by taking the average of the Mean Fluorescence Intensity of 10 fibers that show high GFP expression and have normal morphology. Calculate the \% Maximum GFP Intensity for each fiber by dividing the Mean Florescence Intensity for each fiber by the Maximum GFP Intensity and multiplying by 100 . Tabulate the results of the GFP analysis for each TA muscle as shown in Figure 3E.

3. Studying muscle fiber integrity and myonuclear location by Immunofluorescent labeling of desmin and 4',6-diamidino-2phenylindole (DAPI), respectively.

NOTE: Designate one set of the slides prepared, as above, to study the desmin labeling in the muscle fibers.

1. Fix sections with $2 \%$ paraformaldehyde in PBS for 10 min. Wash the sections three times with PBS.

CAUTION: Wear appropriate personal protective equipment (PPE) when handling when handling paraformaldehyde.

2. Block sections with $3 \%$ bovine serum albumin diluted in PBS containing $0.01 \%$ Triton-X100 for 30 min. This blocking solution is referred to hereafter as the primary diluent.

3. Dilute the rabbit anti-desmin immunoglobulin $\mathrm{G}(\lg G)$ in the primary diluent $(1: 200)$

4. After the blocking is complete, apply the diluted primary antibodies on sections and incubate overnight in a refrigerator at $\sim 4{ }^{\circ} \mathrm{C}$. Wash the sections once ( $5 \mathrm{~min}$ ) with PBS containing $0.1 \%$ Triton X-100. This solution is referred to hereafter as the first wash buffer.

5. Wash the sections 3 times (5 min per wash) with PBS.

6. Prepare the secondary antibody by diluting goat anti rabbit lgG (conjugated to red fluorescent dye) in PBS containing $0.01 \%$ Triton $\mathrm{X}-100$.

7. Apply the diluted secondary antibody on the sections and incubate at room temperature $\left(\sim 23^{\circ} \mathrm{C}\right)$ for $60 \mathrm{~min}$.

8. Wash the sections once ( $5 \mathrm{~min}$ ) with the first wash buffer. Wash the sections 3 times ( 5 min per wash) with PBS.

9. Apply DAPI for $3 \mathrm{~min}$. Wash the sections 3 times (1 min per wash) with PBS. Apply $\sim 50 \mu \mathrm{L}$ of the anti-fade medium on the sections and apply glass coverslip. Seal the coverslip edges with clear nail polish.

10. Using the light and fluorescent microscope (see Table of Materials), study the serial sections with a 10X objective and an appropriate filter for visualizing red fluorescent dye.

11. Collect the digital images $(\sim 15)$ of the overlapping visual fields to cover the entire cross section of the TA muscle. Collect the fluorescence images of DAPI labeling for each of the visual fields by switching to the appropriate filter setting.

12. Open and analyze the images using a suitable imaging software as described in steps 7.2.3 - 7.2.4.

NOTE: Study images to identify, which muscle fibers are damaged (desmin negative), which muscle fibers have central nucleation (desmin positive fibers that have DAPI-labeled nuclei located internally rather than peripherally), and, which areas of the section have inflammation and/or fibrosis (areas that have many DAPI-labeled nuclei clustered together, but are devoid of muscle fibers).

At 3 days post-MIME, the donor EDL that is implanted by MIME is contained within the host TA muscle compartment (Figure 2A-C). As expected, the cross sections of the TA muscle from donor mice, studied under fluorescence optics, do not show green fluorescence because they do not express GFP (Figure 2D). In contrast, the cross sections of the TA muscle from host mice that are not implanted with donor tissue, show uniform green fluorescence in the TA muscle fibers, as the fibers express GFP (Figure 2E). In the cross sections of muscles implanted by MIME with donor muscle tissue, there is a line of demarcation between the host muscle fibers (GFP+) and donor muscle fibers (GFP-). The phase contrast images of the visual fields shown in Figure 2D-F are presented in Figure 2E'-F' and suggest that there are indeed muscle fibers present in the regions where there is no GFP signal.

At 14 days post-MIME and $\mathrm{BaCl}_{2}$ injection, by studying serial cross sections of the TA muscle that are left unlabeled or are labeled with antibodies to desmin (Figure 3), we learn that the GFP signal is expressed by all the muscle fibers in the untreated muscle from host mice, but not in the MIME-treated muscle (red desmin labeling detects viable muscle fibers). In the host TA muscle treated with $\mathrm{MIME}$ and $\mathrm{BaCl} 2$ injection, the donor-cell-derived myogenesis is evident from the presence of many desmin $(+)$ muscle fibers that show no detectable GFP signal (Figure 3C-D, 3 C'-D'). Chimeric muscle fibers arising from the likely fusion of the host and donor myogenic cells can be detected by the low to moderate levels of GFP fluorescence exhibited by these fibers. Numerous chimeric fibers appear across the entire diameter of the TA muscle suggesting that the donor SCs are capable of migrating several hundreds of microns within the epimysium of the host muscle. Quantitation of GFP+ fibers is shown in Figure 3E. Figure 4 shows high magnification images of serial cross sections of an entire $\mathrm{MIME}+\mathrm{BaCl}_{2}$ treated TA muscle. 

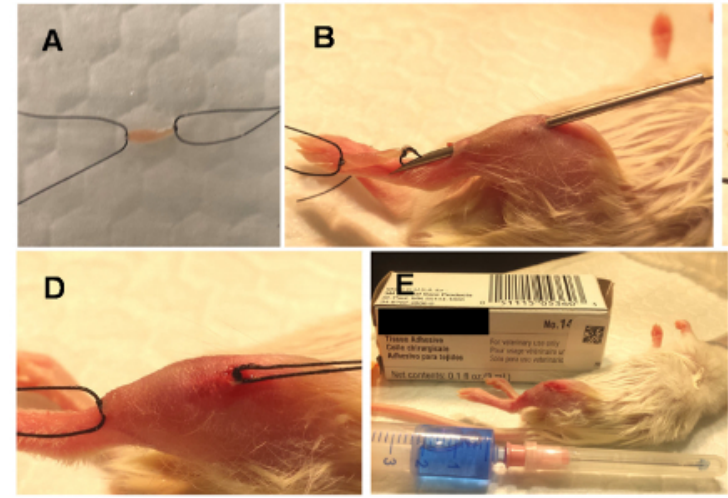

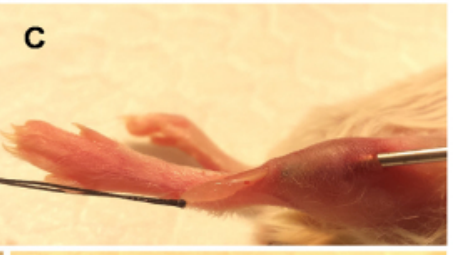

$\mathbf{F}$

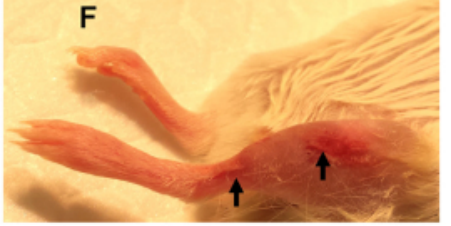

Figure 1. Steps involved in Minimally Invasive Muscle Embedding (MIME).

MIME is performed under general anesthesia. It involves placing $\sim 10 \mathrm{mg}$ of donor muscle (donor mouse EDL) in Ringer solution and tying the guiding sutures to its ends (A). An 18-gauge needle is passed through the long axis of the host muscle (B) and the donor tissue is drawn through the needle track (C). The donor tissue is left embedded in the host muscle (D), the guiding sutures are cut, and the needle holes are sealed with tissue adhesive $(E)$. The sealed needle wounds are indicated with arrows $(F)$. Please click here to view a larger version of this figure.
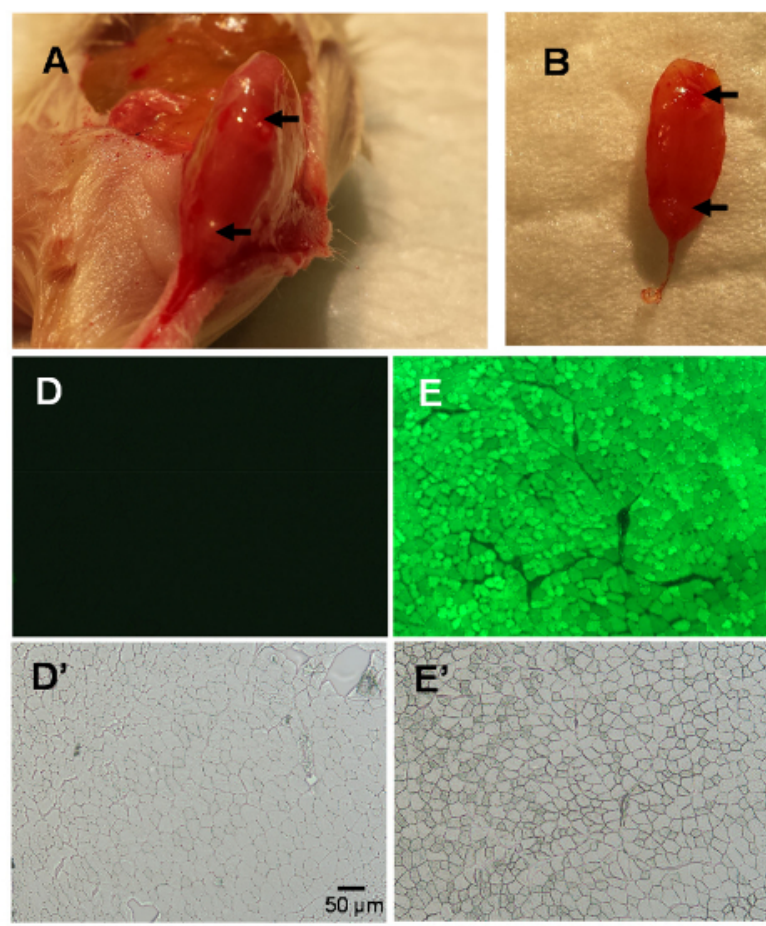
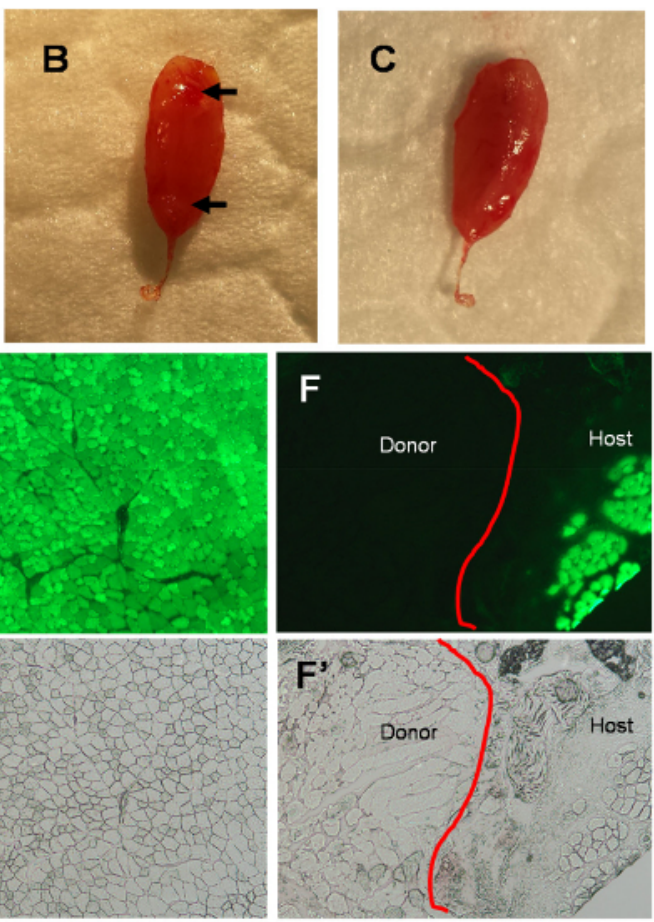

Figure 2. Studies of the TA Muscle 3 Days After MIME.

Host muscle collected 3 days after MIME; note needle marks on host TA (arrows; A-B). Data further confirm that the embedded donor mouse $E D L$ is ensconced within the TA muscle compartment (B-C). Fluorescence images of TA muscle cross sections show no green fluorescence in wild-type TA muscle (D), bright green fluorescence in NSG-GFP TA muscle (E), and a distinction between host and donor muscle in NSG-GFP TA muscle at 3 days post-MIME (F). The phase contrast images of the visual fields in D-F are shown in $D^{\prime}-F^{\prime}$, respectively. The red line in panels $\mathrm{F}$ and $\mathrm{F}^{\prime}$ shows the line of demarcation between the host and donor tissue. Scale bar $=50 \mu \mathrm{m}$. Please click here to view a larger version of this figure. 

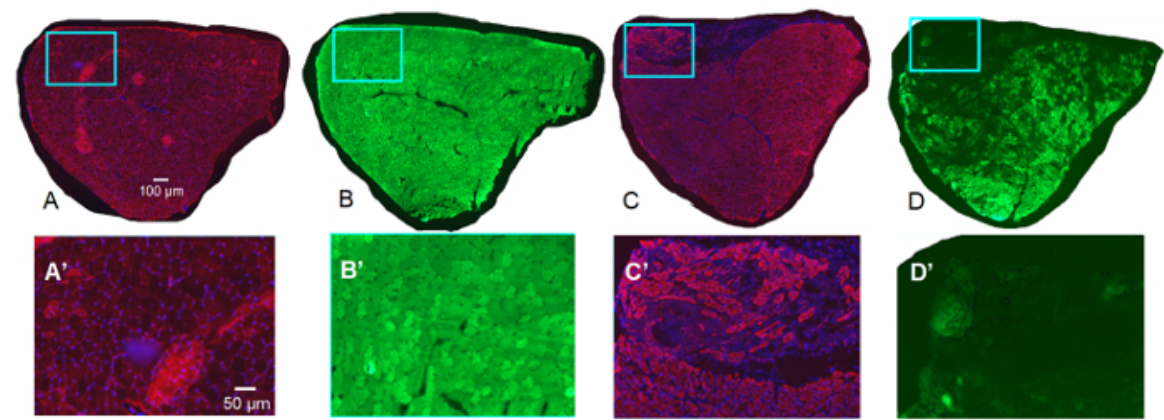

\begin{tabular}{|l|l|l|l|l|l|}
\hline Total Fibers & $\begin{array}{l}\text { Clustered GFP(-) fibers } \\
\text { with < 5\% Max GFP } \\
\text { Intensity }\end{array}$ & $\begin{array}{l}\text { Fibers with 5 - 24 \% } \\
\text { Max GFP Intensity }\end{array}$ & $\begin{array}{l}\text { Fibers with 25 -75\% } \\
\text { Max GFP Intensity }\end{array}$ & $\begin{array}{l}\text { Fibers with }>\text { 75\% } \\
\text { Max GFP Intensity }\end{array}$ & $\begin{array}{l}\text { Fibers with Central } \\
\text { Nucleation }\end{array}$ \\
\hline $\begin{array}{l}\text { Panel C-D; } \\
\text { MiME+BaCl }\end{array}$ & $\begin{array}{l}\text { No detectable host } \\
\text { contribution }\end{array}$ & $\begin{array}{l}\text { Little to no host } \\
\text { contribution }\end{array}$ & $\begin{array}{l}\text { Moderate host } \\
\text { contribution }\end{array}$ & $\begin{array}{l}\text { Little to no donor } \\
\text { contribution }\end{array}$ & Regenerated fibers \\
\hline 2069 & $99(5 \%)$ & $548(26 \%)$ & $539(26 \%)$ & $883(43 \%)$ & $1963(95 \%)$ \\
\hline 2717 & $0(0 \%)$ & $0(0 \%)$ & $512(19 \%)$ & $2201(81 \%)$ & $5(0 \%)$ \\
\hline
\end{tabular}

Figure 3. Studies of TA Muscle 14 Days after MIME.

Data collected 14 days post-MIME are presented. The red signal is from immunofluorescent labeling of desmin (a positive signal for desmin denotes that myofibers are viable), the blue signal is from DAPI (stains nuclei), and the green signal is from GFP. In Control TA muscle (right hindlimb) harvested from the host mouse, as expected, nearly all myofibers are positive for desmin, suggesting that these myofibers are viable (A; red signal). Additionally, based on DAPI staining, it is evident that myonuclei of nearly all myofibers are peripherally located, as would be expected in healthy muscle (A; blue signal). Serial sections of the Control TA muscle show that, nearly all muscle fibers are bright green, implying that they are positive for GFP. In MIME+BaCl 2 -treated TA muscle (left hindlimb), donor-cell-mediated myogenesis is evident from the presence of many desmin(+) muscle fibers that show no detectable GFP fluorescence (C-D, C'-D'). Desmin(+) muscle fibers with low to moderate GFP fluorescence (chimeric muscle fibers) are present across the diameter of the entire TA muscle, suggesting that, MIME provides donor SCs that can migrate within the epimysium of the TA muscle and promote donor-cell-derived myogenesis. A'-D' are high-magnification images of the regions within the blue boxes in A-D. Quantitation of GFP(+) fibers and centrally nucleated fibers are shown in E. Scale bars $=100$ $\mu \mathrm{m}(A-D)$ and $50 \mu \mathrm{m}\left(A^{\prime}-D^{\prime}\right)$. Please click here to view a larger version of this figure.
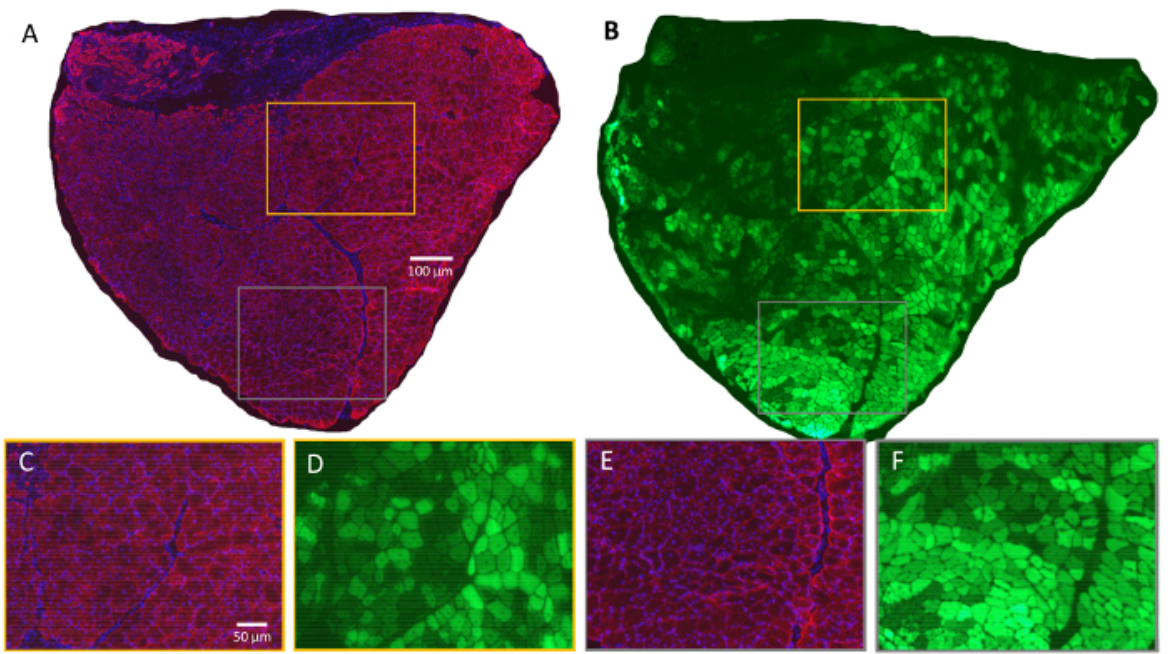

Figure 4. Evidence of Widespread Damage and Regeneration 14 Days after $\mathrm{MIME}$ and $\mathrm{BaCl}_{2}$ Injection.

High magnification, serial cross sections of an entire $\mathrm{MIME}+\mathrm{BaCl}_{2}$ treated TA muscle are presented. The data show that many muscle fibers, which are moderately or strongly GFP+ have centrally located nuclei (A-B; red signal in A is from desmin, blue signal in A is from DAPI, and green signal in $\mathrm{B}$ is from GFP). These data suggest that degeneration and regeneration after $\mathrm{BaCl}_{2}$ injection does not affect GFP expression. In this context, the presence of centrally nucleated muscle fibers in $\mathrm{MIME}+\mathrm{BaCl}_{2}$ treated TA muscle, with little-to-no GFP expression, suggests that these fibers are the likely result of myogenesis (or regeneration) with significant contribution from GFP- myogenic cells. These observations can be further verified in high magnification images of selected fields $(C-F ; C$ and $D$, and $E$ and $F$, respectively, are overlapping regions from serial cross sections; color coding of image borders denote the location of those regions in A and B). Scale bars $=100 \mu \mathrm{m}(\mathbf{A}-\mathbf{B})$ and $50 \mu \mathrm{m}(\mathbf{C}-\mathbf{F})$. Please click here to view a larger version of this figure.

\section{Discussion}

Here, we present a detailed protocol for the novel experimental technique known as MIME, developed in our laboratory, to implant donor muscle tissue into host muscle tissue. This is an adaptation of an open muscle grafting technique that has already proven to be effective in promoting donor-cell-mediated myogenesis in a host muscle ${ }^{9,10,17}$. 
The goal of MIME is not to enable engraftment of the donor muscle tissue itself into the host muscle (we currently do not know if this occurs), but rather to provide a source of donor SCs that can contribute to myogenesis in the host muscle under conditions that stimulate muscle regeneration. Our hope is that after MIME has been optimized and tested in basic and preclinical studies, it could provide valuable insights to guide clinical therapies aimed at increasing muscle regeneration in skeletal muscles that have undergone myogenic muscle loss.

There are numerous questions that have yet to be answered regarding how MIME could be translated into a clinical therapy, for example: How would we control the quality of donor tissue? How would we control immune rejection of donor tissue and cells? Is the donor tissue cleared after providing cells for myogenesis or does it leave behind a fibrotic scar? Does the fiber type of donor and/or host muscle affect the donor-cellmediated myogenesis? Which muscles can practically benefit from MIME? We are currently expanding our studies to assess if MIME is safe and effective, identify adjunctive treatments that can augment donor-derived myogenesis, and answer many of the questions, listed above.

After completing our tests of mouse-to-mouse allogeneic transplantation with MIME, our next step is to perform human-to-mouse MIME with cadaveric human tissue to evaluate the myogenic potential of cadaveric muscle tissue. We anticipate that these experiments will lead to a new line of basic and translational research, which involves muscle tissue from donors, who are registered in initiatives such as the Body Bequest Program for education and the Gift of Life program for organ donation.

The representative data presented in this manuscript suggest that at 14 days after MIME, there are several viable myofibers that either lack GFP or have low levels of GFP. Our interpretation of these data is that GFP- donor satellite cells contributed to the myogenesis in the host muscle. We performed this experiment in preparation for implantation of human cadaveric tissue into a host mouse muscle. To demonstrate unequivocally that the donor satellite cells contribute to myogenesis in the host muscle following MIME, it would be useful to implant donor tissue that expresses a fluorescent reporter, which can be easily distinguished from GFP (e.g., red fluorescent protein expressing donor tissue implanted into GFP+ host muscle).

This technique is mainly limited by the nature of the SCs present in the donor tissue. If the SCs in the donor tissue are viable, the technique is likely to facilitate donor-cell-mediated myogenesis, while if they are not viable, myogenesis cannot occur. However, since SCs are very resilient and are viable for about 2 weeks post-mortem, it is highly likely that for experimental purposes, the donor tissue that is implanted within a few minutes after harvest will facilitate donor-cell-mediated myogenesis ${ }^{13}$. Additionally, as alluded to above, it is possible that since the whole muscle tissue (that contains SCs, mature muscle fibers, as well as muscle-resident fibroblasts) is embedded into the host muscle, fibrosis could occur. However, this assumption needs to be empirically examined. The literature on muscle damage arising from injurious contractions, cryoinjury, and experimental myotoxins, suggests that immune cells (mainly macrophages) are capable of effectively clearing cellular debris from damaged fibers and remodeling the extracellular matrix ${ }^{18}$. Therefore, it is possible that after $\mathrm{MIME}$ and $\mathrm{BaCl}_{2}$ injection, as long as the host immune cells have access to degenerating donor muscle fibers, they could clear debris, leaving behind just the donor SCs. Finally, the extent of the donorderived myogenesis is dependent on the amount of donor muscle tissue that is embedded in the host muscle. In order for the host muscle compartment to be completely repopulated by donor-cell-derived myofibers, it would require a method like X- or gamma-irradiation to ablate host muscle SCs and also require repeated MIME procedures.

It has been demonstrated that surgically exposing a host muscle and suturing a piece of donor tissue onto the host muscle can facilitate donorcell-mediated myogenesis ${ }^{9,10,17}$. This open surgical approach has been used in the past to track the progression of myogenesis and to generate mouse models of human muscle diseases. The innovative aspect of the MIME technique is that it is minimally invasive and does not involve surgically exposing the host muscle. This reduces the risk of iatrogenic infection and the degree of discomfort in the host animal, therefore making it more feasible to perform MIME repeatedly on the same host muscle if needed.

We anticipate that the MIME technique might be a suitable refinement to the open surgical approach that is currently followed to implant donor muscle tissue into a host mouse. This could expedite the generation of humanized mouse models, by embedding biopsied human muscle in the host mouse muscle. Additionally, based on our preliminary data from mouse-to-mouse grafting, we anticipate that MIME will be effective in achieving donor-cell-mediated myogenesis from human cadaveric donor tissue as long as donor SCs are viable. Finally, with additional testing and validation, we hope that the MIME technique will help develop new therapies to facilitate donor-cell-mediated myogenesis in humans with muscle diseases.

The success of the MIME technique is critically dependent upon precisely embedding the donor tissue within the fascial compartment (epimysium) of the host muscle. Only if the donor tissue is placed within the host muscle compartment, will it be able to provide SCs to the host muscle in a precise manner. If the donor tissue is placed outside of the host muscle, it is unclear as to what would be its fate. In our experimental model for MIME, we use the TA muscle as the host muscle, since it is prominent and superficially placed in the anterolateral aspect of the leg. The size, orientation and anatomical position of the TA muscle, makes it easy to confirm that the donor tissue is placed correctly within the host TA muscle after MIME. In this paper, we have provided experimental evidence that the donor tissue remains embedded within the host TA muscle at 3 days post-MIME, and that there is donor-cell-mediated myogenesis in the host muscle at 14 days post-MIME.

\section{Disclosures}

The authors have no competing financial interests.

\section{Acknowledgements}

This work was made possible by a Pilot Grant from the Alliance for Regenerative Rehabilitation Research and Training (AR ${ }^{3} T$ ) and a Faculty Startup Package from Wayne State University to JAR. $\mathrm{AR}^{3} \mathrm{~T}$ is supported by the Eunice Kennedy Shriver National Institute of Child Health and Human Development (NICHD), National Institute of Neurological Disorders and Stroke (NINDS), and National Institute of Biomedical Imaging and Bioengineering (NIBIB) of the National Institutes of Health under Award Number P2CHD086843. The content is solely the responsibility of the authors and does not necessarily represent the official views of the National Institutes of Health. 


\section{References}

1. Mauro, A. Satellite cell of skeletal muscle fibers. J Biophys Biochem Cytol. 9 493-495 (1961)

2. Robertson, T. A., Grounds, M. D., Papadimitriou, J. M. Elucidation of aspects of murine skeletal muscle regeneration using local and whole body irradiation. J Anat. 181 ( Pt 2) 265-276 (1992).

3. Charge, S. B., Rudnicki, M. A. Cellular and molecular regulation of muscle regeneration. Physiol Rev. 84 (1), $209-238$ (2004).

4. Brack, A. S., Rando, T. A. Tissue-specific stem cells: lessons from the skeletal muscle satellite cell. Cell Stem Cell. 10 (5), $504-514$ (2012).

5. Krag, T. O., Hauerslev, S., Sveen, M. L., Schwartz, M., Vissing, J. Level of muscle regeneration in limb-girdle muscular dystrophy type 21 relates to genotype and clinical severity. Skelet Muscle. 1 (1), 31 (2011).

6. Skuk, D., et al. Dystrophin expression in myofibers of Duchenne muscular dystrophy patients following intramuscular injections of normal myogenic cells. Mol Ther. 9 (3), 475-482 (2004).

7. Beauchamp, J. R., Morgan, J. E., Pagel, C. N., Partridge, T. A. Dynamics of myoblast transplantation reveal a discrete minority of precursors with stem cell-like properties as the myogenic source. J Cell Biol. 144 (6), 1113-1122 (1999).

8. Sakellariou, P., et al. Neuromuscular electrical stimulation promotes development in mice of mature human muscle from immortalized human myoblasts. Skelet Muscle. 6 (1), 4 (2015).

9. Collins, C. A., et al. Stem cell function, self-renewal, and behavioral heterogeneity of cells from the adult muscle satellite cell niche. Cell. $\mathbf{1 2 2}$ (2), 289-301 (2005).

10. Zhang, Y., et al. Human skeletal muscle xenograft as a new preclinical model for muscle disorders. Hum Mol Genet. 23 (12), $3180-3188$ (2014).

11. Juhas, M., Engelmayr, G. C., Jr., Fontanella, A. N., Palmer, G. M., Bursac, N. Biomimetic engineered muscle with capacity for vascular integration and functional maturation in vivo. Proc Natl Acad Sci U S A. 111 (15), 5508-5513 (2014).

12. Lin, H., Cheng, A. W., Alexander, P. G., Beck, A. M., Tuan, R. S. Cartilage tissue engineering application of injectable gelatin hydrogel with in situ visible-light-activated gelation capability in both air and aqueous solution. Tissue Eng Part A. 20 (17-18), 2402-2411 (2014).

13. Latil, M., et al. Skeletal muscle stem cells adopt a dormant cell state post mortem and retain regenerative capacity. Nat Commun. 3903 (2012).

14. Maykel, J., et al. NOD-scidll2rg (tm1Wjl) and NOD-Rag1 (null) II2rg (tm1Wjl) : a model for stromal cell-tumor cell interaction for human colon cancer. Dig Dis Sci. 59 (6), 1169-1179 (2014).

15. Casar, J. C., et al. Heparan sulfate proteoglycans are increased during skeletal muscle regeneration: requirement of syndecan-3 for successful fiber formation. J Cell Sci. 117 (Pt 1), 73-84 (2004).

16. Hardy, D., et al. Comparative Study of Injury Models for Studying Muscle Regeneration in Mice. PLoS One. 11 (1), e0147198 (2016).

17. Roberts, P., McGeachie, J. K., Grounds, M. D., Smith, E. R. Initiation and duration of myogenic precursor cell replication in transplants of intact skeletal muscles: an autoradiographic study in mice. Anat Rec. 224 (1), 1-6 (1989).

18. Tidball, J. G. Mechanisms of muscle injury, repair, and regeneration. Compr Physiol. 1 (4), 2029-2062 (2011). 